\title{
Good MRI images: to Gad or not to Gad?
}

\author{
Henning Steen • Vedat Schwenger
}

Received: 26 February 2007 / Revised: 8 April 2007 / Accepted: 11 April 2007 / Published online: 22 May 2007

(C) IPNA 2007

\begin{abstract}
Gadolinium-based magnetic resonance imaging (MRI) contrast agents (Gad-CA) were formerly considered as alternatives to X-ray-employed iodinated media. Although originally thought to be nonnephrotoxic and proven to be nonhazardous in a healthy population, the Gad-CA safety issue is progressively more controversial in the high-risk group of end-stage renal disease (ESRD) patients. Recently, Gad-CAs have not only been blamed for harmless side effects such as dizziness or nausea but also for much more severe complications such as acute renal failure, pancreatitis, or even the development of so-called "nephrogenic systemic fibrosis" in patients with renal failure, culminating in the prohibition of gadodiamide (Omniscan) administration in ESRD patients and, due to renal-organ immaturity, in newborns and infants up to 1 year old. This editorial is written to give insights into the molecular structure of Gad-CAs as well as into the potential biochemical pathomechanisms underlying the aforementioned severe clinical manifestations. Furthermore, a review about the latest literature on Gad-CA nephrotoxicity is provided. Potential risk factors are mentioned and strategies to avoid deterioration of renal function are presented. Cases
\end{abstract}

\footnotetext{
H. Steen

Department of Cardiology, University Hospital Heidelberg, Im Neuenheimer Feld 410,

69120 Heidelberg, Germany

V. Schwenger

Department of Nephrology, University Hospital Heidelberg, Im Neuenheimer Feld 162,

69120 Heidelberg, Germany

V. Schwenger $(\bowtie)$

Medizinische Universitätsklinik Heidelberg,

Im Neuenheimer Feld 162,

69120 Heidelberg, Germany

e-mail: Vedat_Schwenger@med.uni-heidelberg.de
}

with Gad-CA-associated adverse events should be adequately documented and reported appropriately. MRI professionals should collaborate closely with their colleagues from other medical specialties to identify patients with adverse events.

Keywords Magnetic resonance imaging .

Gadolinium contrast agents · Side effects of gadolinium .

Acute renal failure (ARF) .

Nephrogenic systemic fibrosis (NSF) .

End-stage renal disease (ESRD)

As nephrologists and colleagues working with magnetic resonance imaging (MRI), we generally take for granted the relative safety of gadolinium contrast agents (Gad-CA) in terms of their nonadverse effects on our patients. On 7 February 2007, the medical community was informed that gadodiamide (Omniscan) is now not only contraindicated in patients with end-stage renal disease (ESRD), but also in pre- or postoperative liver transplantation patients and, due to their renal-organ immaturity, in newborns and infants up to 1 year old. This change in policy was motivated by recent reports of a possible link between the use of gadodiamide and the development of so-called "nephrogenic systemic fibrosis" (NSF) [1-4] in patients with renal failure. This discovery came as quite a surprise to the nephrology and radiology communities.

A glimpse into medical literature reveals approximately 200 cases of NSF, previously known as nephrogenic fibrosing dermopathy (NFD) [1-6]. One pediatric case is published in this issue [7]. NSF is characterized clinically by the thickening, induration, and hardening of the skin in the distal extremities, followed by the skin of the trunk but usually omitting the face [8]. The diagnosis of NSF is confirmed by skin biopsy. Characteristically, histopathologic features such as thickened collagen bundles with surround- 
ing clefts, mucin deposition, and proliferation of fibroblasts and elastic fibers are present, whereas signs of inflammation are absent, which makes this disorder a distinct entity $[2,8]$. Grobner [8] was the first to report that five of nine gadodiamide-exposed hemodialysis patients developed NSF within 2-4 weeks after contrast-dye exposure. A systemic process is assumed in which circulating fibrocytes of bone marrow origin are aberrantly recruited to various body sites, including the skin, likely triggered or exacerbated by Gadinduced endothelial damage [9]. Following this report, Marckmann [10] also described 13 cases of NSF in patients exposed to gadodiamide, progressing in more than $50 \%$ of the cases to severe disability and even one patient death.

To date, there are eight Gad-based contrast media available in Europe: gadobenate-dimeglumine (Multihance, Bracco Diagnostics, Inc.), gadobutrol (Gadovist, Schering), gadodiamide (Omniscan, GE Healthcare), gadofosveset (Vasovist, Schering), gadopentetate-dimeglumine (Magnevist, Schering), gadoteric acid (Dotarem, Guerbet), gadoteridol (ProHance, Bracco Diagnostics Inc.), and gadoxetic acid disodium (Primovist, Schering). In each product, the Gad ion is chelated to various anion groups to form Gad salts [11].

The mechanism by which some Gad-CAs might trigger NSF is still unresolved; however, several theories have been proposed. Gad-CAs have different molecular structures that affect their biochemical properties. For example, Omniscan and OptiMARK have a linear and uncharged molecular configuration with excess chelate, which seems to be more likely to release free gadolinium ions $(\mathrm{Gd} 3+)$ into the body, a process called transmetallation. Linear and charged (e.g., Magnevist, MultiHance, Primovist, and Vasovist) as well as cyclic and uncharged (e.g., Gadovist and ProHance) Gad-CAs seem to be less likely to release free $\mathrm{Gd} 3+$ into the body. Dotarem, for example, has a molecular charge and a cyclical structure and is least known for transmetallation. However, the exact mechanisms by which free gadolinium ions can stimulate NSF is unknown and therefore under investigation. Most of the approximately 200 cases of NSF that were reviewed so far in the literature have been associated with the agents Gadodiamide (Omniscan) and Gadoversetamide (OptiMARK, Mallinckrodt), which is not licensed for use in the European Union (EU) but is available in the USA. A small number of cases have been associated with Gadopentetate Dimeglumine (Magnevist, Schering), and to date, no cases of NSF have been associated with other gadolinium-containing contrast agents.

These alarming reports give rise to a nagging question: Is this just the tip of the iceberg? Have clinicians overlooked other possible side effects of Gad? The widespread reliance on Gad due to its nontoxicity probably needs to be reexamined. These MRI contrast agents were originally introduced as alternatives to iodinated media; iodine contrast dyes have become the third most common cause of acute renal failure. In patients with preexisting renal insufficiency, the incidence was as high as $50 \%$ [6]. Although originally thought to be nonnephrotoxic, Gad-based contrast media have recently been implicated in various other critical incidents (apart from $\mathrm{NSF}$ ) in patients with ESRD. Again, a glance at the literature reveals that colleagues have been studying Gad-contrastinduced nephrotoxicity (CIN) since the early 1990s. CIN is now defined by the European Society of Urogenital Radiology (ESUR) [12] as an increase in serum creatinine of more than $25 \%$, or more than $0.5 \mathrm{mg} / \mathrm{dl}$, occurring within 3 days after the intravascular administration of the contrast medium in the absence of an alternative etiology. Interestingly, the controversial results are mostly due to the differing definitions of nephrotoxicity, patient's collectives, renal diseases, contrast agents, and contrast-agent concentrations that have been employed and published. Unfortunately, there are almost no published data on pediatric patients with renal impairment.

As early as 1992, Haustein et al. [13] reported that GadDiethylene triamine pentaacetic acid (DTPA) $[0.1 \mathrm{mmol} / \mathrm{kg}$ body weight (bw)] was harmless in a study of 21 patients with chronic renal failure (creatinine clearance $34.5 \mathrm{ml} / \mathrm{min}$ ). Ten years later, Rieger et al. [5] described only one episode of acute renal failure in 39 Gad-DTPA $(0.35 \mathrm{mmol} / \mathrm{kg}$ bw $)$ angiographic imaging procedures for 29 patients $(59 \%$ of whom were diabetic) with renal insufficiency (mean serum creatinine: $3.6 \mathrm{mg} / \mathrm{dl}$ ). However, recent reports paint a different picture. Erley et al. [14] recorded a significant decline of renal function in 11 of 21 patients with severe renal impairment (mean creatinine clearance $31 \mathrm{ml} / \mathrm{min}$ ) after gadobutrol $(>0.5 \mathrm{mmol} / \mathrm{kg})$ digital subtraction angiography. In a larger study, Sam et al. [15] comprehensively investigated 195 patients with chronic renal insufficiency (creatinine clearance $38 \mathrm{ml} / \mathrm{min}$ ) who were subjected to Gad angiography $(0.25 \mathrm{mmol} / \mathrm{kg}$ bw or more $)$ and found that acute renal failure developed in seven patients (3.5\%). Serum creatinine returned to baseline levels within 4-12 days in five patients, but ESRD developed in two patients. In our own collective of 50 patients with pre-ESRD or ESRD referred for cardiovascular MRI dobutamine stress echo test, and myocardial perfusion/viability, we observed a marked systemic inflammatory response signalled by increased inflammatory markers and fever up to $40^{\circ} \mathrm{C}$ and partial hypereosinophilia in seven patients $24 \mathrm{~h}$ after Gad-DTPA administration $(0.3 \mathrm{mmol} / \mathrm{kg} \mathrm{bw})$. Acute renal failure was experienced in two patients, one of whom did not recover.

Do we know what mechanisms lead to renal impairment or systemic reaction after gad exposure? Unfortunately, due to sparse data on the renal lesions associated with this condition, very little is known about the mechanism of Gad nephrotoxicity. The nephrotoxic effects of iodinated contrast agents, which have been studied more intensely, are 
multifactorial, including a vasoconstrictive effect leading to hypoxic or ischemic tubular cell injury and a direct tubulotoxicity mediated by the generation of reactive oxygen species [12]. As Gad-based and iodinated contrast agents share the same pharmacodynamics $[12,15]$ and their nephrotoxic effects are often clinically similar, they may cause renal damage through the same mechanisms. The mechanism of the severe inflammatory reaction is akin to a massive allergic reaction, but the exact pathomechanism is poorly understood. Initiation of dialysis may promptly relieve the symptoms. Recently, Akgun [16] published a renal biopsy study documenting a reversible acute tubular cell injury in which patchy tubular cell necrosis and degeneration along with mild interstitial edema and inflammation were observed. The patient had been doubly exposed to Gad in the forms of Gad-DTPA and gadopentetate. No significant glomerular or vascular changes were observed.

Gad chelates are distributed in the extracellular space and are eliminated almost exclusively by the kidney through glomerular filtration. Renal failure impairs but maintains Gad excretion without resorting to a nonrenal route. For example, gadodiamide half-life is $1.3 \mathrm{~h}, 34.3 \mathrm{~h}$, and $52.7 \mathrm{~h}$ in healthy patients, patients with ESRD, and peritoneal dialysis patients, respectively [17]. This extremely long half-life raises the question of how to handle pediatric patients on peritoneal dialysis (PD). Should they be hemodialyzed after Gad exposure, or should Gad exposure be completely avoided in PD patients? Further data are necessary to determine an optimal form of treatment.

What other potential systemic Gad effects could be responsible for the severe adverse events? Gadodiamide, for example, leaves two to four times more Gad in the bone than other contrast agents in patients with normal renal function $[10,12]$. Because of the longer half-life of Gad-based contrast media in patients with ESRD, it could be speculated that Gad liberation might be causing systemic effects.

Taken together, these issues confront us with the task of correctly identifying and preparing our renal failure patientsand especially pediatric patients - with ESRD who were frequently treated with PD. At the same time, we must protect them from an unjustified risk caused by the possibility of adverse Gad-associated events. Raised serum creatinine levels (particularly secondary to diabetic nephropathy), dehydration, congestive heart failure, an age of over 70 years, and concurrent administration of nephrotoxic drugs (nonsteroidal anti-inflammatory drugs) are all considered potential risk factors by the guidelines [12]. Consequently, patients undergoing a diagnostic MRI scan with potential Gad exposure should be well hydrated, low- or iso-osmolar contrast media should be used, and nephrotoxic drugs should have been stopped for at least $24 \mathrm{~h}$. Alternatively, other imaging techniques that do not require the administration of
Gad should be taken into consideration. All in all, the less Gad, the better! In patients on continuous ambulatory PD (CAPD) and severely reduced renal function, one should avoid Gad doses exceeding $0.3 \mathrm{mmol} / \mathrm{kg}$ bw [12].

The treatment of Gad-induced nephropathy begins with recognizing the condition. Therefore, in high-risk patients, the measurement of serum creatinine (a threshold of $1.5 \mathrm{mg} / \mathrm{dl}$ may be the ideal level for Gad agents, as is common with iodinated contrast agents) between the second and fourth day post-MRI will identify the nonaffected patients. Additionally, patients should not be reexposed to Gad before the kidney function has returned to its previous state. If $\mathrm{Gad}$ contrast media has to be administered again, the patients should at least be adequately hydrated and nephrotoxic agents should be stopped in due time.

Prompt hemodialysis within the first several hours after MRI may be recommended for quick removal of the Gad agent from the bloodstream. MRI professionals should collaborate closely with their colleagues from other medical specialties in the search to identify patients with Gadassociated adverse events. Cases with a positive history of exposure to Gad and adverse events should be adequately documented and reported through the appropriate channels. More data are required to clarify whether these complications are limited to one specific MRI contrast agent or might also occur with other types of Gad contrast media.

In conclusion, compelling evidence challenges the safety record of Gad contrast agents with respect to the conditions for which they were rather indiscriminately used in the past. We have to admit that the "carefree" days-when many of us thought that we at last had one imaging modality wherein contrast agents could be used nearly risk-free in renal-failure patients - are gone.

\section{References}

1. Cowper SE, Robin HS, Steinberg SM, Su LD, Gupta S, LeBoit PE (2000) Scleromyxoedema-like cutaneous diseases in renal-dialysis patients [research letter]. Lancet 356:1000-1001

2. Cowper SE, Su LD, Bhawan J, Robin HS, LeBoit PE (2001) Nephrogenic fibrosing dermopathy. Am J Dermatopathol 23:383-393

3. Ting WW, Stone MS, Madison KC, Kurtz K (2003) Nephrogenic fibrosing dermopathy with systemic involvement. Arch Dermatol 139:903-906

4. LeBoit PE (2003) What nephrogenic fibrosing dermopathy might be. Arch Dermatol 139:928-930

5. Rieger J, Sitter T, Toepfer M, Linsenmaier U, Pfeifer KJ, Schiffl H (2002) Gadolinium as an alternative contrast agent for diagnostic and interventional angiographic procedures in patients with impaired renal function. Nephrol Dial Transplant 17:824-828

6. Solomon R (1998) Contrast-medium-induced acute renal failure. Kidney Int 53:230-242

7. Dharnidharka VR, Wesson SK, Fennell RS (2006) Gadolinium and nephrogenic fibrosing dermopathy in pediatric patients. Pediatr Nephrol. DOI 10.1007/s00467-006-0384-Z 
8. Grobner $T$ (2006) Gadolinium - a specific trigger for the development of nephrogenic fibrosing dermopathy and nephrogenic systemic fibrosis? Nephrol Dial Transplant 21:1104-1108

9. Cowper SE, Boyer PJ (2006) Nephrogenic systemic fibrosis: an update. Curr Rheumatol Rep 8:151-157

10. Marckmann P, Skov L, Rossen K, Dupont A, Damholt MB, Heaf JG, Thomsen HS (2006) Nephrogenic systemic fibrosis: suspected causative role of gadodiamide used for contrast-enhanced magnetic resonance imaging. J Am Soc Nephrol 17:2359-2362

11. Spinosa DJ, Kaufmann JA, Hartwell GD (2002) Gadolinium chelates in angiography and interventional radiology: a useful alternative to iodinated contrast media for angiography. Radiology 223:319-325

12. Thomsen HS, Morcos SK (2003) Contrast media and the kidney: European Society of Urogenital Radiology (ESUR) guidelines. Br J Radiol 76:513-518
13. Haustein J, Niendorf HP, Krestin G, Louton T, SchuhmannGiampieri G, Clauss W, Junge W (1992) Renal tolerance of gadolinium DTPA/dimeglumine in patients with chronic renal failure. Invest Radiol 27:153-156

14. Erley CM, Bader BD, Berger ED (2004) Gadolinium-based contrast media compared with iodinated media for digital subtraction angiography in azotaemic patients. Nephrol Dial Transplant 19:2526-2531

15. Sam AD 2nd, Morasch MD, Collins J, Song G, Chen R, Pereles FS (2003) Safety of gadolinium contrast angiography in patients with chronic renal insufficiency. J Vasc Surg 38:313-318

16. Akgun H, Gonlusen G, Cartwright J, Suki WN, Truong LD (2006) Are Gadolinium-Based Contrast Media Nephrotoxic? A Renal Biopsy Study. Arch Pathol Lab Med 130:1354-1357

17. Rosenthal DI, Becerra CR, Toto RD, Carbone DP, Frenkel EP (2000) Reversible renal toxicity resulting from high single doses of the new radiosensitizer gadolinium texaphyrin. Am J Clin Oncol 23:593-598 